\title{
MARTÍNEZ, M., ZAMORANO, A. (COORDS.) (2018). TEORÍA Y METODOLOGÍA PARA LA ENSEÑANZA DE ELE. EDITORIAL EN CLAVE-ELE: ESPAÑA
}

\author{
María Gabriela Amador Solano \\ Instituto Tecnológico de Costa Rica \\ gamador@itcr.ac.cr \\ https://orcid.org/0000-0001-5027-1480
}

El libro que se reseña corresponde a una colección de cuatro volúmenes bajo el título Teoría y metodología para la enseñanza de ELE. Los ejes temáticos de cada uno de los volúmenes reúnen un panorama completo de los fundamentos teóricos, las vinculaciones e interrelaciones de las teorías de adquisición de lenguas. Es una obra que se recomienda para la formación de docentes en la enseñanza del español como lengua extranjera (ELE), pues permite una clara reflexión en el ejercicio profesional de todo educador, ante las exigencias del siglo XXI.

Los coordinadores y editores de la obra son María Martínez-Atienza y Alfonso Zamorano Aguilar, ambos profesores titulares de la Universidad de Córdoba (España). Su vasta experiencia en la enseñanza de una segunda lengua les permitió dirigir esta obra y escribir un sinnúmero de publicaciones relacionadas con la enseñanza de ELE. La colección de volúmenes se organiza en los siguientes ejes temáticos: Fundamentos, enfoques y tendencias (volumen 1), Enseñanza-aprendizaje de los componentes lingüísticos (volumen 2), Programación y diseño de unidades didácticas (volumen 3) y Literatura, cine y otras manifestaciones culturales (volumen 4).

Cada libro cuenta con una secuencia pedagógica completa y con rigor metodológico: fundamentos teóricos de cada tema, propuestas didácticas para desarrollar en el aula de ELE, pruebas e instrumentos de evaluación, temas para la reflexión y ampliación, así como una selecta bibliografía comentada.

En el volumen 1, se incluyen cuatro capítulos: La ASL desde una aproximación psicolingüística, las teorías lingüísticas como fundamento de los enfoques y métodos en la enseñanza de ELE, metodologías en la enseñanza-aprendizaje de segundas lenguas, el MCER y el Plan curricular del IC. Todos han sido escritos por profesores de la Universidad de Córdoba, especialistas en las áreas de lingüística y filología. Sus líneas de investigación les han permitido publicar libros, artículos de revistas, capítulos, o bien ser parte de grupos de investigación inscritos en la misma universidad.

Para citar esta reseña / To cite this book review: Amador Solano, María Gabriela (2020). Reseña de Martínez, M., Zamorano, A. (Coords.) (2018). Teoría y metodología para la enseñanza de ELE. Editorial En Clave-ELE: España, 2018. ELUA, 34: 215-219. https://doi.org/10.14198/ELUA2020.34.10

Enlace / Link: https://doi.org/10.14198/ELUA2020.34.10 
En primer lugar, se plantea la importancia de realizar investigaciones recientes en torno a los cambios que han tenido lugar a través de la historia, la adquisición de segundas lenguas. Quienes realizan investigación deben ser capaces de explicar este fenómeno tanto si tiene lugar en el contexto de una segunda lengua, como si es en el de una lengua extranjera. A lo largo de un proceso investigativo de tres décadas, se destacan aclaraciones terminológicas que permiten el estudio de procedimientos más adecuados para la mejora continua de la enseñanza de la L2 en un contexto académico y formal, estas son: el lenguaje como habilidad cognitiva, definición entre adquisición de L1 y adquisición de L2, la interlengua, así como el aprendizaje y enseñanza de segundas lenguas.

El desarrollo de tales procedimientos requiere un tratamiento metodológico basado en la adopción de premisas teóricas coherentes que fundamenten la aproximación al proceso psicolingüístico. Se exponen los presupuestos epistemológicos de la ciencia cognitiva a partir de la adquisición de una segunda lengua (ASL). Estudios como el método BRaIN en el ámbito de la enseñanza instrumental para adultos, entre otras investigaciones que han contribuido en este campo, requieren de una serie de disciplinas para lograr la evidencia en el resultado esperado. A modo de reflexión, es importante una revisión profunda de los procesos formativos dirigidos a los futuros docentes con el fin de incluir esta perspectiva interdisciplinar.

En segundo lugar, se demuestra a través de ejemplos precisos que la teoría lingüística dominante en cada momento histórico ha propiciado o condicionado los métodos de enseñanza de segundas lenguas que se usan en la didáctica de la adquisición de un idioma. Se realiza una exposición de algunas de las corrientes lingüísticas que han ido dando sustento a los diversos métodos y enfoques en la enseñanza de las lenguas extranjeras, tales como: la gramática tradicional, el estructuralismo, las repercusiones de la lingüística cognitiva en la enseñanza de lenguas, la irrupción de las funciones comunicativas en la enseñanza de una L2. De esta manera, queda demostrado que todas estas presentan un sustrato de ideas e hipótesis acerca de los rasgos constitutivos del lenguaje humano y han inspirado estrategias de enseñanza-aprendizaje de una segunda lengua de acuerdo con los principios que estas mismas corrientes defienden.

En tercer lugar, para que una enseñanza se pueda calificar de comunicativa debe mostrar una atención integradora en la que el objetivo principal sea preparar al alumno para explotar de forma óptima una competencia comunicativa en un contexto real de aprendizaje. No obstante, el libro nos permite reflexionar sobre los factores que influyen en la calidad de este tipo de comunicación. No obstante, dependerá de la lengua dominante, de la lengua materna, de la motivación del alumnado, de la propia motivación del profesorado o de la efectividad de las estrategias, técnicas y actividades propuestas. Asimismo, se dan una serie de sugerencias didácticas como el enfoque por tareas, que sirven para estructurar y poner en práctica el uso del lenguaje como una herramienta utilizada para conseguir un propósito comunicativo.

Finalmente, en el primer volumen se expone, por un lado, el Marco Común Europeo de Referencia (MCER), el cual surge de la necesidad de estandarizar los sistemas de evaluación y certificación de las distintas lenguas europeas. En este documento se presenta un planteamiento de actuación dual: las dimensiones vertical y horizontal. La vertical es la encargada de estructurar y secuenciar las distintas etapas del progreso del estudiante de lengua; la horizontal, engloba las distintas categorías descriptivas del uso de la lengua, así como los fenómenos que intervienen en ella. Por otro lado, el Plan Curricular del Instituto Cervantes (PCIC) se basa en las normas establecidas por el MCER para proporcionar al 
educador un amplio repertorio de materiales de carácter lingüístico-comunicativo, que le permitan diseñar las unidades didácticas para la enseñanza en el aula.

Por su parte, el volumen 2 está dedicado a la enseñanza de los componentes lingüísticos $\mathrm{y}$ ha sido escrito por distintos especialistas que exponen de manera profesional tanto la fundamentación teórica, como las propuestas didácticas de los diversos temas, organizadas de acuerdo con la secuencia de desarrollo establecida en el PCIC y que toman en consideración las recomendaciones del $M C E R$, del Consejo de Europa. Oportunamente, le sigue un apartado dedicado a la evaluación de los conocimientos y habilidades relativos al tema de estudio. Se incluyen cuatro capítulos sobre enseñanza-aprendizaje en distintos componentes: Fonético-fonológico, Gramatical, Léxico-semántico y Pragmático.

El capítulo 1 ofrece una descripción exhaustiva del componente fonético-fonológico y expone detalladamente los fenómenos segmentales y suprasegmentales. Para poder entender estos conceptos, se desarrolla cada uno de los tres tipos de información: lingüística, paralingüística y extralingüística. Quienes enseñan español deben tener en cuenta estos tres niveles de información y entrenar a los aprendientes para reconocer y producir los patrones principales que sirven de vehículo a la transmisión de significados.

Tanto el MCER como el PCIC incluyen con objetivos muy claros el nivel fónico que habrá que tener en cuenta en la didáctica, a la vez que incluyen los estándares de aprendizaje de la lengua española que deben conseguirse en cada nivel (A1-2, B1-2, C1-2). De ahí que este capítulo destaque la importancia de repasar temas como: el plano fónico de la lengua, la base articulatoria, el inventario de fonemas del español, el nivel suprasegmental (la sílaba, el acento y la entonación), el plano sintagmático y el paradigmático. Merece la pena que el docente de ELE incluya estos contenidos en sus clases y siga las estrategias didácticas que se le sugieren con el uso de la tecnología: uso de laboratorios de idiomas, grabaciones de audio y vídeo, entre otras.

El capítulo 2 expone las aportaciones que la investigación ha realizado en los últimos dos decenios en relación, en primer lugar, con la función del componente gramatical en la enseñanza de ELE; en segundo término, con los procedimientos metodológicos más oportunos para su incorporación al aula de idiomas. La enseñanza del componente gramatical se ha alejado de los formatos tradicionales orientados a la producción y basados en práctica mecánica, significativa y libre, esto para dar paso al modelo de análisis de AF, el cual presenta la gramática como una tarea de respuesta a un problema comunicativo en un contexto concreto, permite que los aprendices cuenten con el mayor número de posibilidades para comunicarse y al mismo tiempo, que completen su formación en este campo.

En este apartado podemos notar que la producción en estos últimos veinte años sobre la gramática para profesores ELE ha sido muy prolífica y de una excelente calidad. Se mencionan una serie de autores que a través de los años nos han aportado un magnífico panorama de la cuestión. Investigadores como López García, Alonso Raya, Llopis García, Castañeda Castro han escrito desde 2005 hasta 2014, textos sobre qué gramática enseñar, explicaciones gramaticales adaptadas a los distintos niveles de referencia para las lenguas, en el contexto de las variantes metodológicas de la atención a la forma.

El capítulo 3 proporciona un panorama completo a través de una reflexión en torno a la complejidad del conocimiento léxico, su adquisición y almacenamiento, así como estrategias, criterios y recursos para la creación de materiales centrados en el vocabulario. Hay un señalamiento importante hacia las tres dimensiones del léxico: la lingüística, la 
psicolingüística y la pedagógica. En este sentido, se analiza qué vocabulario existe en los actos comunicativos, qué es lo que sucede en la mente de los aprendientes y de qué modo se enseña en un contexto de aprendizaje determinado.

Las últimas investigaciones en este campo sugieren que la enseñanza del léxico se dé por medio de grupos temáticos según el contenido sobre el que versa el aprendizaje en el aula. Es fundamental conocer qué es lo que saben nuestros aprendientes, qué necesitan saber y cuál es el método de instrucción más eficaz. En el apartado de "Cuestiones para la reflexión" se plantea que los educandos deben tener más que simplemente un entendimiento superficial del significado de una palabra, deberían desarrollar una representación del significado rica y específica, así como un conocimiento de los rasgos formales de la palabra y su funcionamiento sintáctico.

Finalmente, el capítulo 4 nos convence sobre la implicación que tiene el componente pragmático en la formación integral del alumnado, dado que el discente debe desenvolverse en situaciones comunicativas reales, debe ajustar estratégicamente sus intervenciones a los fines comunicativos, tomando en consideración los valores sociales y culturales por los que se rige la comunidad. Dos de los subtemas que se mencionan son: la teoría de los actos de habla y la teoría de la cortesía verbal, ambos dependen de la variabilidad cultural en la que se desarrollan, es decir, varían de una cultura a otra y son una fuente habitual de malentendidos interétnicos.

El volumen 3 se titula "Programación y diseño de unidades didácticas" e incluye cuatro capítulos: Diseño de unidades didácticas, la evaluación en la enseñanza del español como segunda lengua, las nuevas tecnologías en ELE y estrategias en la enseñanza-aprendizaje de la lengua y la literatura en ELE, el componente afectivo. Los autores realizan un largo recorrido sobre la presencia y variedades de evaluación en unos 40 manuales de éxito publicados en los últimos diez años. Al final de cada capítulo se expone una bibliografía comentada de gran utilidad para comprender mejor el objetivo de cada apartado.

En el primer capítulo, los autores abordan de una manera exhaustiva, ordenada y sistemática lo que se puede considerar la molécula de la organización docente, es decir, la unidad didáctica. Se exponen los términos: enfoque, método, currículo, programa y enfoque por tareas, así como diferentes planteamientos para el diseño de una lección y con ello, la confección de materiales didácticos. Respecto a este último, es necesario que las actividades que se realicen potencien un uso autónomo, que habitúen a la inferencia de reglas y que propicien la toma de conciencia de los procesos que albergan.

En el segundo capítulo, los autores realizan un largo recorrido sobre la presencia y variedades de evaluación en manuales publicados sobre español para extranjeros, los cuales integran cantidad de actividades de diversa índole, pero casi ninguno precisa sobre cuáles son los criterios de evaluación en cada caso. Por eso los docentes tienen el compromiso de manejar este tema en función de las características de las actividades. En este sentido, podremos comprobar que el MCER y el PCIC nos orientan con líneas básicas sobre el modo de llevar a cabo este proceso. Se termina con algunos temas de reflexión, en especial se rescata que la evaluación sea formativa, dado que la información generada debe servir para la toma de decisiones.

En el tercer capítulo, se expone un resumen de la gran cantidad de recursos que las nuevas tecnologías ponen en manos del docente de ELE. Los clasifican en tres grupos: los dispositivos informáticos, las aplicaciones informáticas y la web. Las TIC se han convertido en una ayuda esencial en todo proceso de enseñanza y aprendizaje, por eso es fundamental que los educadores inviertan su tiempo en nuevas metodologías que las incluyan. Este apartado 
nos da ese panorama de todos los recursos disponibles de forma organizada y coherente, sin embargo se propone la tarea de crear una base de datos actualizable, un repositorio general de recursos TIC en ELE, con el que el docente pueda trabajar.

En el cuarto capítulo, el tema principal es la actitud personal con la que se enfrenta el estudiante en el proceso de aprendizaje. En este sentido, el PCIC propone un inventario de "saberes y comportamientos socioculturales" en el que se describen aspectos sobre las relaciones interpersonales, identidad colectiva y estilo de vida, así como "habilidades y actitudes interculturales" necesarias para afrontar con éxito el proceso de adquisición de una segunda lengua. Por otro lado, aparece un conjunto de propuestas didácticas y sugerencias pedagógicas que prestan atención al cómo, qué, con qué, para el diseño de técnicas que fomenten la toma de conciencia, el desarrollo de la autonomía y el aprendizaje reflexivo.

Finalmente, el volumen 4 se titula "Literatura, cine y otras manifestaciones culturales", e incluye tres capítulos de distinto alcance y de diferentes contenidos y objetivos: Enseñanzaaprendizaje del componente cultural, la literatura en ELE y el cine en la clase de ELE. La persona interesada en este libro encontrará contenido actualizado, planteamiento de problemas y propuestas de solución, modelos de actividades y ejercicios con los cuales trabajar en clase y pautas para la reflexión autónoma. Tal como que en los otros volúmenes, al final de cada capítulo se expone una bibliografía comentada de gran utilidad para comprender mejor el objetivo de cada apartado.

En el capítulo 1 se ofrece un repaso de las principales definiciones del término cultura y la importancia de esta sensibilización cuando se aprende una segunda lengua. Se analizan en este apartado proposiciones como: Cuál es la necesidad real de enseñar cultura en el aula de ELE, qué problemas puede plantear el enseñar la cultura del español, qué significa enseñar una L2 sin enseñar su cultura o cómo se evalúa este componente. Asimismo, se nos presenta un planteamiento de cómo enseñar este tema en el aula desde tres categorías: Cultura con mayúsculas, Cultura a secas y Kultura con K. Gracias a esta propuesta de Miguel y Sans (2004), se ha producido en los últimos años un giro radical en la didáctica de esta área de estudio, pues se enseña inmersa en cualquier acto de habla, lejos de los estereotipos.

El capítulo 2 nos hace reflexionar sobre las múltiples maneras de incorporar la literatura al aula de ELE y sobre la necesidad de elegir adecuadamente el material para el correcto desarrollo de los objetivos de aprendizaje. La literatura en el aula de ELE exige del profesor un trabajo continuado de búsqueda y elaboración de materiales, selección de textos y adecuación didáctica de las tareas en función de las posibilidades de aprendizaje de los estudiantes. Al final del capítulo se ofrecen algunos temas de reflexión: tener en cuenta las características de los distintos géneros literarios cuando se diseñe el programa docente, usar el taller literario para estimular el uso creativo del lenguaje en el aula y asignar a los educandos dos fragmentos con su respectivo análisis de comprensión de lectura.

Para finalizar el libro, se ofrece en el capítulo 3 de este cuarto volumen un tema que rescata la importancia de la cultura visual, esto es el estudio del cine como instrumento de gran valor para desarrollar un amplio abanico de competencias: comunicativa, cultural, intercultural y pragmática, entre otras. Asimismo, se exponen los medios audiovisuales aplicados a la enseñanza y el aprendizaje de segundas lenguas en los programas de formación de profesores. Nos queda claro después de la lectura que la integración del cine en el aula de ELE favorece un enfoque interdisciplinario y transversal, potencia el trabajo colaborativo, a la vez que contribuye al conocimiento de este campo como industria cultural y económica. 